eelworms in nursery stock, in flower crops and in carrots. Against root knot eelworm under glass, ethylene dibromide at twice the usual dosage of $45-60 \mathrm{~cm} .{ }^{3} / \mathrm{m} .^{2}$ gave good results. Some of the newer products, while showing approximate killing efficiency, had the disadvantage of remaining phytotoxic for six weeks or more after application-a serious handicap in horticultural soils where a quick succession of cropping is essential. The use of sodium methyl dithiocarbamate, a good general soil sterilizer against eelworms and fungi, was described by Messrs. Linden and Schicke (Ingelheim), who showed that mixing-in or injection at the rate of $100 \mathrm{~cm} \cdot{ }^{3} / \mathrm{m}^{2}$ are both preferable to watering-in. They recognize the phytotoxicity risk, and recommend that soil heaps in the open air should be treated in summer as early as possible before use ; if left until November it is not safe to use the soil until March. Lettuce is very susceptible to injury by this sterilizer and is therefore a good indicator of the time for safe planting. Eelworm attack on carrots has been recognized as a puzzling problem; the species of eelworm concerned was described only as recently as 1949 by Thorne as Hoplolaimus uniformis. Soil treatment with $\mathrm{DD}$ and with formalin gave a high kill of this eelworm, and was followed by markedly improved growth. Other studies by Kuiper and Drijfhout (Netherlands) showed that, in infested soil, crop rotation influenced yield; the yield of carrots following potatoes proved to be better than that following either sugar-beet or carrots.

The beet eelworm survey in northern France was described by Dr. M. Christmann, of the Institut Technique de la Betterave, Paris. He showed that the Institute can handle up to two thousand soil samples per year and use its accumulating knowledge of the rate of decrease in viable cysts, in particular regions and localities, as the basis for its advisory work. Another contribution from France was an interesting paper by Dr. F. Chaboussou, director of the Institute of Agricultural Zoology of the Gironde. He described investigations on the plum codlin moth, Laspeyresia funebrana-a most troublesome pest throughout western Europe, and one that is outstandingly difficult to control. Spraying of plums with oleoparathion in early July when 75-80 per cent of the caterpillars were hatched, but before the fourth instar was reached, gave a total kill of more than 90 per cent with a single treatment. It was noted that the fourth and fifth instars are not easily reached as they are deep in the fruits. There is, in practice, a period of about eight days during which spraying can be very effective.

Three speakers presented papers on the subject of spider mites. J. Meltzer showed that ovicides, such as CPBS and CPCBS, applied to the upper surface of bean leaves, are capable of killing red spider eggs laid afterwards on the lower surfaces, and eggs already laid there may also be killed as well as a high proportion of the immature stages. With tedion, which penetrates the leaf more slowly, the toxic action lasts for as long as three weeks. R. Delhaye has been investigating the use of emulsions containing phosphoric ester, white oil and ammonium thioricinate and claimed good results against red spider on vines and peaches under glass. M. van de Vrie's observation on the strawberry tarsonemid mite greatly interested his audience. He emphasized the difficulty of controlling, in the field, mites the development of which takes place largely in the very young unfolded leaves in the crown. The esters of phosphoric acid are not really satisfactory in this case, and endrin and kelthane, though active for a long period through their toxic residues, are not desirable on fruiting beds because of their high mammalian toxicity. Mr. J. Bernard, of Gembloux, on the other hand, was concerned with trying to secure mammalian toxicity-in control of the field vole, Microtus arvalis. He found that while red squill is not satisfactory, coumaphene in baits with crushed oats or flour is more effective, but this species of vole is more resistant to this poison than the laboratory white rat.

Other subjects that occupied the conference were soil disinfection, antibiotics for use on horticultural crops, fungus control in pine nurseries, seed disinfection of flax, the aerial fungus flora, exobasidium of the azalea, a crop of great importance in Belgium particularly, and the bacterial canker of poplar, a crop tree of great economic value from the Netherlands right down to Italy. Herbicides, too, received attention, and particularly those for use in strawberry beds, in nurseries, and in flax crops.

In the tropical section of the symposium, P. L. G. Benoit dealt with the distribution and behaviour of flies the larve of which cause myiasis in man and animals in the Belgian Congo. A. Gillard and Prof. J. van den Brande described the recent collection of new species of Heterodera-type eelworms in the Congo and directed attention to their economic importance, and Prof. P. A. van der Laan discussed the use of the insecticide toxaphene and emphasized its advantages under tropical conditions. The control of rice pests, and notably of the injurious molluses Pomacea lineata and $P$. glauca, was described by J. van Dinther, who obtained good results against these molluses with applications of either copper sulphate or BHC.

The organizers of the symposium can be congratulated on its success, and the participants-and indeed the much wider public vitally concerned with the subjects covered-will look forward to the early publication of the papers in full. H. W. MILES

\section{SOME RECENT ADVANCES IN SPECTROSCOPY}

A

CONFERENCE on spectroscopy, held in the Department of Chemistry in the University of Western Australia during February 20-21, was attended by about forty persons, including a number of visitors from the eastern States. The meeting was opened by Dr. A. L. G. Rees (Division of Industrial Chemistry, Commonwealth Scientific and Industrial Research Organization), who surveyed recent developments in the field of spectroscopy. The major theoretical advance in recent years has undoubtedly been the introduction by Bayliss of the free electron approximation in quantum mechanics and its subsequent development, mainly by American workers. New techniques of special importance are the magnetic methods based on nuclear and electron spin resonance which can provide useful chemical information. Other notable advances in technique are echelle spectroscopy, due to Harrison, the high-intensity Raman source developed by Welsh at 'Toronto, and the development of multiple-pass spectroscopy and a method of analysis based on atomic absorption spectra, both due to Walsh and his colleagues. Also of importance are the developments by Watanabe 
and Williams of direct-reading vacuum ultra-violet spectroscopy and of electron-impact spectroscopy by Morrison. Important new observations include the measurement of rotational Raman spectra, the discovery of intermolecular combination bands, and the discovery by Bayliss and Brackenridge of spurious bands in solution spectra near the edge of intense solvent absorption bands. Outstanding problems are associated with the general unsatisfactory state of quantum-mechanical calculations; new and improved methods are badly needed even for simple molecules. Moreover, the problems of the computing of intensities of infra-red and Raman spectra and the determination of the causes of solvent shifts of absorption spectra are as yet unsolved. Many other important problems are associated with the Raman excitation, absorption and emission of solids and the investigation of unexplored regions in the very far infra-red.

The remainder of the first session was devoted to research contributions. Dr. A. R. H. Cole (Department of Chemistry, University of Western Australia) discussed the infra-red spectra and stereochemistry of cyclohexane derivatives. No simple method is available for determining the preferred conformations of di- and tri-substituted cyclohexanes in which there may be competition between substituents as to which will occupy the more stable equatorial position. Dr. Cole described the infra-red absorption due to free and hydrogen-bonded (intramolecular) hydroxyl groups in a series of cyclohexane-1,2-diols, each of which carries one or two other substituents of different sizes in the axial $(a)$ and/or equatorial $(e)$ position. A large group such as isopropyl can act as an anchor holding a cyclohexane ring in one conformation ( (e)-isopropyl) and can prevent hydrogenbonding between a trans-1,2-diol system by keeping the hydroxyl groups in the diaxial form. 1-Ethylcyclohexane-trans-1,2-diol exists as an equilibrium mixture of $(e)$-ethyl-(a)-diol and $(a)$-ethyl-(e)-diol, and intensity measurements of free and bonded hydroxyl absorption carried out by Miss G. T. A. Muller show the mixture to contain 35 per cent of the former. 1-Methylcyclohexane-trans-1,2-diol, on the other hand, exists completely in the $(a)$-methyl(e)-diol conformation which allows relatively strong hydrogen-bonding. 'The measurements were extended to a series of $p$-menthane diols, and it was shown that at room temperature trans-2-hydroxyneoisomenthol exists as an equilibrium mixture containing 68.5 per cent of the $(a)$-methyl- $(a)$-diol- $(e)$-isopropyl conformation. From this it can be readily deduced that the conformation of neaisomenthol is $(a)$-methyl-(a)hydroxyl-(e)-isopropyl. Lowering of the hydroxyl stretching frequency due to hydrogen-bond formation can be related to the conformation and in suitable cases can be used to determine configurations of cyclohexane-1,2-diols.

Mr. A. Walsh (Division of Industrial Chemistry, Commonwealth Scientific and Industrial Research Organization) described the development with Dr. N. S. Ham of a new Raman source. Methods for obtaining the necessary intensity for Raman spectra studies invariably make use of the mercury arc. The equipment is often very bulky, requiring the use of either several mercury lamps-the light being concentrated by multiple reflexion-or of water-cooled ares drawing as much as 50 amp. (Toronto source). The design of the Melbourne source was also stimulated by the need for a source of longer wave-length for the study of the vibration frequencies of the iodine molecule in solution. The new source, which was demonstrated, is electrodeless and consists essentially of an annular jacket containing a rare gas and an alkali metal or mercury. For excitation a high. frequency diathermy source $(2,450 \mathrm{Mc}$./s.) provides extremely intense illumination. By using ciesium as the alkali metal it is hoped to obtain a Raman source of wave-length 8521 A. Very few molecules absorb appreciably in this region.

Prof. N. S. Bayliss (Department of Chemistry, University of Western Australia) followed with an account of studies on the effects of solvents on infra. red intensities carried out in collaboration with Dr. A. R. H. Cole and Mr. L. H. Little. In this work the intensities of the $\mathrm{C}=\mathrm{O}$ stretching frequency in acetone and other ketones, of the $\mathrm{C} \equiv \mathrm{N}$ stretching frequency in a series of aliphatic and aromatic nitriles, and of the $\mathrm{S}-\mathrm{H}$ stretching frequency in several mercaptans were all measured in a series of solvents with a wide range of dielectric constants. All the spectra show changes of intensity with change of solvent, the change being particularly large for the $\mathrm{C} \equiv \mathrm{N}$ and the $\mathrm{S}-\mathrm{H}$ bands. The nitrile intensities, for example, are more than twice as large in chloroform as in isooctane, while the mercaptan intensities are eight times as large in pyridine as in isooctane. The general trend observed was that intensities increase with the dielectric constant of the solvent, but there are marked individual exceptions to this rule. The $\mathrm{C} \equiv \mathrm{N}$ intensity for benzonitrile is in all cases about four times as large as for acetonitrile in the same solvent. A puzzling feature is that the $\mathrm{C} \equiv \mathrm{N}$ intensity for phthalonitrile, with two nitrile groups, is only about one-half the intensity for benzonitrile with one nitrile group. No current theory is capable of explaining the experimental results. The classical theories of Chako and of Schuyer are far from reproducing the intensity changes, and while the quantum theory of Hirota is rather closer, it still does not account for the large intensity variations observed. There is also no adequate theoretical explanation of the fact that the half-width of the bands observed in solution is in all cases considerably less than the halfwidth of the same bands observed in the gas. Current quantum theory of infra-red intensities relates the intensity to the change of bond moment with internuclear distance. The problem is to determine how this quantity is affected by the solvent environment.

The second session commenced with a paper by Dr. A. L. G. Rees on the theory of solvent effects. To account for solvent effects on spectra it is formally necessary to set up the wave equation of the whole assembly of particles, both solute and solvent, in the liquid. In view of the impossibility of solving such an equation, it is convenient to approximate by considering a single solute molecule surrounded by $\mathbf{a}$ nearest-neighbour shell of $z$ solvent molecules. There are experimental grounds for believing that the influence of more remote solvent molecules can be neglected to this degree of approximation. In terms of the Franck-Condon principle, the molecular environment is static during an optical transition. In general, the nearest-neighbour environment of a particular solute molecule is unsymmetrical. The perturbation due to the environment can be expressed in terms of three components, which are respectively independent, and even and odd functions, of the co-ordinates. The application of first-order perturbation theory then makes it possible to set up a formal expression for the transition moment in solution. A significant result is that not only 
transitions which are allowed in the free molecule will be altered in intensity, but also that transitions which are totally forbidden in the free molecule will acquire a first-order transition moment in solution owing to the operation of the unsymmetrical part of the perturbation. This general theory can be applied to the intense ultra-violet band shown by halogens in aromatic hydrocarbon solutions. Although this band has been attributed by Mulliken to complexes, the present theory suggests that it is more likely to be associated with a known totally forbidden transition in the free molecule.

Mr. C. J. Brackenridge (Department of Chemistry, University of Western Australia) then described changes in the ultra-violet spectrum of $s$-trinitrobenzene with the composition of seven binary solvent mixtures all containing aniline and covering the complete composition range. Aniline was chosen because of its notable effect in enhancing the absorption in the $4000 \mathrm{~A}$. region. When the results were plotted in accordance with Scott's rearrangement of the Ketelaar equation, which predicts a straight line for 1 : 1 soluto-solvent complex formation, and from which the equilibrium constant can be determined, an interesting effect was observed. Points of discontinuity appeared in the vicinity of 0.5 aniline mole fraction in all seven systems showing that, although linear plots could be drawn in several cases on both sides of the point, they are incompatible with the postulate of complex formation. In the two systems hexanol-aniline and ethylene glycol-aniline, the effect of temperature on the $s$-trinitrobenzene spectrum was studied. The heat of formation was calculated on the highly improbable assumption that one of the plots on either side of the point represented solvation. In both systems the $H$ values were low and their sign changed on either side of the point of discontinuity. It is suggested that the point of discontinuity indicates a molecular rearrangement arising in the course of a physical perturbation.

The session concluded with a description by Mr. J. V. Sullivan (Division of Industrial Chemistry, Commonwealth Scientific and Industrial Research Organization) of his investigation of the causes of certain anomalies in the spectrum of iodine. 'These anomalies are lack of reproducibility of the molar extinction coefficients, $\varepsilon$, high values of the coefficients in the region from 4000 to $2200 \mathrm{~A}$., and a sharp rise in the spectral curve from approximately $\varepsilon=100$ to $\varepsilon=600$ in the region $2100-2000 \mathrm{~A}$. Furthermore, a shift of the order of 500 kaysers, generally towards longer wave-lengths, has been observed in the peak of the curve in the visible region. Extensive investigation now shows that the anomalies are very probably due to the formation of an adsorbed iodine layer on the end-windows of the cell. Experiments on cells made up with internal windows, which allow greater concentrations of the adsorbed layer, have shown the expected increase in the molar extinction coefficients.

The final session of the Conference commenced with a paper by Mr. A. Walsh describing the analytical advantages of absorption spectroscopy and the technique of measuring atomic absorption spectra. Analyses by emission spectra lack sensitivity since even at very high temperatures only a small proportion of atoms are excited to emission, while almost all of the atoms are potential absorbers. Measurements of absorption spectra, which are free from complications such as inter-element effects, self-absorption and self-reversal, therefore have many advantages. Rather than use a continuous source, it is more feasible to measure the absorption coefficient at the centre of the line using a sharp-line source such as a hollow-cathode discharge tube. Modulation of the incident radiation is necessary to avoid recording radiation emitted by the vaporized sample. By using copper as standard, it has been possible to determine approximately the oscillator strengths of a number of elements. While the method has proved successful for the analysis of many elements, some, such as aluminium, are not atomized in a flame. Samples have been vaporized both from solutions sprayed into a flame, and from alloys by a sputtering discharge through a rare gas. In the latter case inter-element effects are present, but the method is likely to prove useful both for analysis and for the study of sputtering behaviour. Several optical variants of the apparatus used were described.

The Conference concluded with the presentation of two papers from the Department of Physies of the University of Western Australia. Mr. R. S. Crisp described a grazing-incidence high-vacuum spectrometer which had been constructed specifically for the study of the soft X-ray band emission spectra of metals in the range $50-1000 \mathrm{~A}$. The spoctrometer utilizes two interchangeable ruled aluminium gratings with 570 and 1,150 grooves $/ \mathrm{mm}$. The diffracted, focused radiation is detected by an Allen-type, open, beryllium-copper photo-multiplier and attached analyser slit, which are mechanically driven around the Rowland circle. The output pulses from the multiplier are amplified and counted on a counting rate meter ; a continuous record of detected intensity is given by a recording potentiometer as the analyser slit traverses the spectrum. In such an instrument the resolution achieved depends not only on the optical resolution of the combination of grating and slits, but also on the statistical deviation and response-time of the counting rate meter and the speed of traverse of the spectrum. A certain minimum speed is fixed by considerations involving long- and short-term contamination of the specimen surface and consequent modification of the observed band shape. A study has been made of the variation of contamination of specimens with time by observing the fall in intensity at a particular wave-length as the contamination proceeds. Spectra were shown which had been obtained for the metals, magnesiam, aluminium, lithium, sodium, potassium, beryllium and copper. 'The 'roll-over' on the high-energy side of the peak of the lithium-potassium spectrum, which was observed by Skinner and queried by Schiff, and Jones and Schiff, has been indisputably observed. The 'emission edges' observed by Gyorgy and Harvey for the $M$-spectrum of copper, which were not observed by Skinner, Bullen and Johnson, also were not observed with the present instrument. Further investigations will be directed towards the determination of the changes in band shapes and band widths and of edge widths and edge shifts in the spectra of the components of solid solutions.

Mr. M. R. Meharry described work on the luminescence of the alkali halides irradiated in the vacuum ultra-violet. The variation in fluoreseence efficiency versus exciting wave-lengths for $\operatorname{som} \Theta \mathrm{KCl}: \mathrm{Tl}$ and KI : Tl phosphors was studied in the wave-length region 900-2300 A. At long wave-lengths, this was found to be correlated closely with the transmission spectrum of the pure crystal. This was considered evidence that only direct excitation of the $\mathrm{Tl}+$ centres occurred and that no exciton-induced fluorescence 
took place. A fluorescence growth under steady excitation and a phosphorescence decay in the dark were observed at wave-lengths less than those corresponding to the series limit for the respective crystals. This was interpreted in terms of a series of trapping and excited energy-levels in $\mathrm{Tl}^{2+}$ as proposed by Johnson and Williams. A series of experiments on photoconductivity gave negative, though not necessarily conclusive, results at wave-lengths less than that generally accepted for the series limit. Also, an observed sharp rise in fluorescence of $\mathrm{KI}: \mathrm{Tl}$ at $1000 \mathrm{~A}$. remained unexplained. It was proposed that, in order to explain the latter results, the ionization limits for $\mathrm{KCl}: \mathrm{Tl}$ and $\mathrm{KI}: \mathrm{Tl}$ must be situated at much shorter wave-lengths than the accepted wave-lengths of $1300 \mathrm{~A}$. and $1750 \mathrm{~A}$., respectively. G. A. Elliotr

\section{COLLOQUE A.M.P.E.R.E., 1957}

THE 1957 meeting of the Colloque A.M.P.E.R.E. (Atomes et Molécules par Etudes Radio-Elec. triques) was held at St. Malo during April 24-26. The conference, which is held annually, is primarily concerned with the study of the properties of materials with the use of radio-froquency electromagnetic waves. The subjects discussed were dielectrics, electron paramagnetic resonance, ferromagnetic resonance, microwave spectroscopy of gases, nuclear magnetic resonance, and quadrupole magnetic resonance. The meeting was attended by some two hundred scientists, the majority being from French laboratories, but with visitors from most European countries, including East Germany and Poland and some from as far as Venezuela and Australia. The organizers of the Conference take pride in the fact that the proceedings are conducted almost entirely in French without apparent effort on the part of the foreigners present.

Some seventy papers were presented mainly in the form of ten minute summaries, preprints being available beforehand. This rather heavy programme, combined with splendid weather and French hospitality, made it rather difficult to follow the programme as closely as one might have wished. Prof. R. Freymann, who is the leading spirit of the Colloque A.M.P.E.R.E., was probably tho only person both to attend every session and be at a banquet every evening. Such energy bodes well for French science.

It is evidently impracticable to report each paper and I shall pick out those which I hope will be representative of the Conference. The papers are published in book-form by the Archives des Sciences, and are available from the Institut de Physique, Université de Genève, price 25 Swiss francs. The discussion at the Conference is not recorded or published, as it is feared that this would tend to suppress a free and frank exchange of views.

The session on dielectrics was opened by Prof. $\mathrm{H}$. Fröhlich (Liverpool), who discussed under what conditions trapped electrons at vacancies in ionic crystals would result in dielectric loss of the Debye type. He showed that this may occur, even for single stationary vacancies, when electron lattice interactions are included, whereas previously it has usually been supposed that moving vacancies which simulate a rotating dipole are necessary. S. Dryden (Sydney) reported recent work on Debye-type dielec- tric absorption in ionic crystals, which they have now found in eleven simple ionic crystals and tentatively associate with diffusion of lattice vacancies. M. J. Meinnel and R. Clinet (Rennes) discussed the dielectric effects of the transitions in the ammonium halides, and demonstrated the absolute necessity of removing all traces of water. F. Lasbleis et al. (Rennes) presented results on dielectric absorption and phase changes in a series of titanates. J. Meinnel and F. Trigolet (Rennes) reported dielectric measurements on hexagonal selenium; they find a Debye absorption which is displaced by suitable irradiation, the effect depending on its intensity and wave-length. Prof. R. Freymann (Rennes) reported on various projects, including dielectric measurements on thiourea and on semi-conductors containing various impurities.

Prof. R. H. Cole (Brown University) presented a survey of his dielectric measurements on liquid alkyl halides and mixtures of these with other organic liquids, in the supercooled state. For the mixtures one sometimes finds one dispersion region and sometimes the original two, for which no satisfactory explanation is at present available. J. Ph. Poley and A. J. van Eick (The Hague) presented further results on the two dispersion regions in liquid chloroand bromo-benzenes. L. de Broukère and M. Mandel (Brussels) suggested a method of explaining the spectrum of dielectric relaxation times in high polymers which is based on a consideration of freedom of motion of chain segments. F. C. de Ronde (Eindhoven) proposed a new method of measuring electric and magnetic constants of solids in the microwave region. C. Brot and A. Soulard (Paris) described a special resonant cavity for the frequency range $100-1,000 \mathrm{Mc} / \mathrm{s}$. which appears to facilitate measurements in this difficult region.

In the sessions on electron paramagnetic resonance there were several papers on free radicals. G. Berthet (Fontenay) has studied some stable free radicals, particularly with regard to the hyperfine structure. Some new radicals have been discovered by Y. Fellion and J. Uebersfeld (Paris). F. W. Heineken and F. M. Schimmel (Amsterdam) find that some stable free radicals may be sensitive to oxygen, just like induced ones. M. Bruma (Paris) described a ferrite circulator which seems to have advantages over the 'magic $T$ ' and similar devices used in paramagnetic resonance equipment. D. J. E. Ingram and J. F. Gibson (Southampton) gave results for radicals formed by ultra-violet irradiation of glassforming substances at low temperatures. D. $\mathrm{H}$. Whiffen et al. (Birmingham) have studied a number of polymers under gamma-irradiation and J. S. van Wieringen (Eindhoven) has investigated glasses subjected to several types of irradiation. At the moment the interpretation of these effects is rather uncertain. D. J. E. Ingram (Southampton) described how structural data on large molecules may be obtained from electron resonance in favourable cases, for example, with hæmoglobin. J. Uebersfeld and E. Erb (Paris) reported further work on paramagnetic centres in carbon. E. E. Schneider (Newcastle) reported further results on alkali halides doped with manganese and on $\mathrm{X}$-irradiated lithium fluoride. A. Gozzini et al. (Pisa) have measured magnetic birefringence effects using DPPH.

The ferromagnetic resonance in a single crystal of iron has been studied by A. Strub (Grenoble). J. Munier and P. Sermet (Grenoble) have developed a ferrite microwave attenuator with 'electronic' control. 